\title{
Die EU als zivil-militärischer Krisenmanager: zwischen Anspruch und Wirklichkeit
}

\author{
Hans-Georg Ehrhart*
}

Die Jahre 1999/2000 markieren eine wichtige Etappe in der Geschichte der Europäischen Union. Im Juni 1999 erklärte der Europäische Rat von Köln, der Union die Fähigkeit und die Mittel zu autonomem Handeln im Bereich der Krisenverhütung und Konfliktbewältigung an die Hand zu geben, um im Einklang mit den Prinzipien der Charta der Vereinten Nationen auf internationaler Ebene zu Frieden und Sicherheit beizutragen. Sechs Monate später erfolgte die Festlegung des Helsinki Headline Goal, das militärische Planziele definierte, die bis 2003 umgesetzt werden sollten. Es folgten Beschlüsse zur besseren Koordinierung nichtmilitärischer Fähigkeiten und zum Aufbau neuer politisch-militärischer Strukturen. Damit beschritten die Europäische Union und ihre Mitgliedstaaten einen Weg, der die Union als zivil-militärischen Akteur im internationalen Krisenmanagement etablieren sollte.

Im Folgenden soll der Frage nachgegangen werden, wie die Union als Krisenmanager funktioniert. Dabei sollen nicht die Aktivitäten in den Krisengebieten im Vordergrund stehen oder die damit verbundenen außenpolitischen Interessen und Leitbilder, sondern die Komplexität des Entscheidungsprozesses. Nach einer kurzen Problematisierung des Akteursstatus wird ausführlicher auf Strukturen, Prozesse und Probleme des Krisenmanagements eingegangen. Dann werden einige Reformvorhaben und -vorschläge angesprochen, um abschließend die Perspektiven der Union als Krisenmanager zu skizzieren.

\section{Ein seltsamer Akteur}

Von der Europäischen Union als Krisenmanager zu sprechen ist nicht unproblematisch. So könnte gefragt werden, wer dieser sicherheitspolitische Akteur eigentlich ist. Die übliche Antwort darauf lautet, dass es sich um einen Akteur sui generis handele. Die Union sei weder ein Staat noch eine internationale Organisation, weniger als eine Föderation, aber mehr als eine Konföderation. ${ }^{1}$ Sie ist als erste wirklich postmoderne Einheit in den internationalen Beziehungen ${ }^{2}$ bezeichnet worden oder als komplexes Mehrebenensystem. ${ }^{3}$ Ihr außen- und sicherheitspolitisches Wirken wird mit so unterschiedlichen Konzepten wie dem einer Zivilmacht, einer Militärmacht oder einer Friedensmacht erfasst. ${ }^{4}$ Weitgehend unstrittig ist, dass

* Dr. Hans-Georg Ehrhart, stellvertretender Leiter des Zentrums für Europäische Friedens- und Sicherheitsstudien und Wissenschaftlicher Referent am Institut für Friedensforschung und Sicherheitspolitik an der Universität Hamburg. Ich bedanke mich bei Gérard Heckel und Hans-Bernhardt Weisserth für wertvolle Hinweise.

1 Vgl. Roy Ginsberg: Conceptualizing the European Union as an International Actor: Narrowing the Theoretical Capability-Expectation Gap, in: Journal of Common Market Studies 3/1999, S. 429-545. Das Bundesverfassungsgericht spricht von einem Staatenverbund, Heinrich Schneider von einer ,starken Konföderation“ oder einer ,,polity sui generis“. Vgl. Heinrich Schneider: Die Zukunft der differenzierten Integration in der Perspektive des Verfassungsvertrages und der Erweiterung, in: integration 4/2004, S. 259-273, hier S. 259.

2 Vgl. John G. Ruggie: Territoriality and Beyond: Problematizising Modernity in International Relations, in: International Organization 1/1993, S. 139-174, hier S. 150.

3 Vgl. Markus Jachtenfuchs/Beate Kohler-Koch: Regieren und Institutionenbildung, in: Markus Jachtenfuchs/ Beate Kohler-Koch (Hrsg.): Europäische Integration, Opladen ${ }^{2} 2003$, S. 11-46.

4 Vgl. Hans-Georg Ehrhart: Die EU und das Leitbild „Friedensmacht“: außen- und sicherheitspolitische Konzepte nach dem Irak-Krieg, in: Thomas Jäger/Alexander Höse/Kai Oppermann (Hrsg.): Transatlantische Beziehungen, Wiesbaden 2005 (im Erscheinen). 
sie in den internationalen Beziehungen ,präsent“ ist. ${ }^{5}$ Des Weiteren besteht Einigkeit darü-

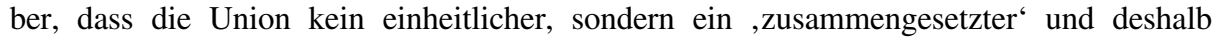
schwerfälliger Akteur ist. ${ }^{6}$

Das trifft insbesondere auf den Bereich der Außen-, Sicherheits- und Verteidigungspolitik zu, in dem die Mitgliedstaaten eifersüchtig ihre Prärogative hüten und nur zu oft ihre nationalen Egoismen pflegen, obwohl sie wissen müssten, dass ihr sicherheitspolitisches Gewicht im Verbund ungleich größer wäre. Daran anknüpfend lautet ein Einwand, vergangene Fehlschläge wie das Desaster im Vorfeld des Irak-Krieges belegten die bremsende, wenn nicht destruktive Rolle nationaler Egoismen und zeigten, dass sich die Union letztlich nicht zu einem veritablen sicherheitspolitischen Akteur entwickeln könne. Dem kann entgegengehalten werden, dass ihre sicherheitspolitische Entwicklung in einem insgesamt positiven Licht erscheint, wenn sie mit der Lage vor Einführung der Gemeinsamen Außen- und Sicherheitspolitik (GASP) und der Europäischen Sicherheits- und Verteidigungspolitik (ESVP) verglichen wird. Die EU-Mitglieder haben seither Erfahrungen gesammelt, ausgewertet und zur Grundlage der weiteren Entwicklung gemacht werden.

Verschiedene Realitäten wirken in diesem langfristigen Prozess als Triebkräfte. Erstens sind die europäischen Verteidigungshaushalte in der letzten Dekade gesunken und werden auf absehbare Zeit auch nicht signifikant steigen. Das eigentliche Problem liegt allerdings nicht in der Höhe der Ausgaben, sondern in der Art und Weise, wie die vorhandenen Gelder ausgegeben werden. Zum einen wird noch immer mehr in traditionelle militärische Sicherheitsvorsorge investiert als in zivile und vorbeugende Maßnahmen. Zum anderen fließt auch innerhalb der Verteidigungsbudgets zu viel Geld in den Erhalt inadäquater Strukturen. Zweitens muss sich die Europäische Union künftig zunehmend selbst um die Sicherheitsbelange des Kontinents und seiner Ränder kümmern. Sie muss folglich ihre Kohäsion so stärken, dass sie in der Lage ist, das öffentliche Gut Sicherheit zu garantieren. Dabei ist sie mit einer einfachen Gleichung konfrontiert: Entweder es gelingt ihr, Stabilität über ihre Grenzen hinaus zu exportieren, oder sie wird zwangsläufig Instabilität importieren. Drittens wird sie auch auf globaler Ebene verstärkt als sicherheitspolitischer Akteur nachgefragt, wobei sie unterschiedlichen Anforderungen gerecht werden muss. Sie reichen im militärischen Bereich von deeskalierenden Maßnahmen über stabilisierende Fähigkeiten bis hin zu Einsätzen mit hoher Intensität und im nichtmilitärischen Bereich von Polizeieinsätzen und Unterstützungsmaßnahmen beim Aufbau von Justiz- und Verwaltungsstrukturen über Katastrophenhilfe bis zu entwicklungspolitischen Maßnahmen und humanitärer Hilfe. Die wichtigste Realität ergibt sich jedoch, viertens, aus den veränderten Risiken und Bedrohungen für Europa im 21. Jahrhundert. Auf absehbare Zeit droht keine militärische Aggression wie zu Zeiten des Ost-West-Konflikts. Die heutigen Kernrisiken resultieren - abgesehen von globalen Herausforderungen wie Klimawandel, Pandemien und Armut - vor allem aus Prozessen des Staatszerfalls, regionaler Instabilität, organisierter Kriminalität, terroristischen Aktivitäten und der Proliferation von Massenvernichtungswaffen. ${ }^{7}$ Aus diesen Risiken ergeben sich drei Konsequenzen für die künftige Sicherheitspolitik: Erstens sind die Staaten der Union mehr denn je auf interne und internationale Zusammenarbeit angewiesen, wenn sie ihre Sicherheit gewährleisten wollen. Zweitens spielen militärische Mittel bei der mittel- und langfristigen Auseinandersetzung mit diesen Risiken eine untergeordnete Rolle. Drittens wird es wird im-

5 David Allan/Michael Smith: The European Union's Security Presence: Barrier, Facilitator, or Manager?, in: Carolyn Rhodes (Hrsg.): The European Union in the World Community, London 1998, S. 45-63.

6 Vgl. Helen Wallace/William Wallace (Hrsg.): Policy-Making in the European Union, Oxford ${ }^{4} 2000$.

7 Vgl. European Council: A secure Europe in a better world. European Security Strategy, Brüssel 2003. 
mer wichtiger, die verschiedenen Instrumente zur Konfliktbearbeitung ressortübergreifend zu vernetzen, und zwar auf nationaler wie auf europäischer Ebene sowie zwischen den beiden.

\section{Zivil-militärisches Krisenmanagement der Europäischen Union}

Die Union sieht sich zu Recht als ein besonderer Akteur im internationalen Krisenmanagement, denn sie verfügt über eine einzigartige Vielfalt an diplomatischen, handelspolitischen, entwicklungspolitischen, justiziellen, polizeilichen und militärischen Instrumenten. Es ist weitgehend unbestritten, dass sich die Krisen des 21 . Jahrhunderts durch große Komplexität auszeichnen. Ihre konstruktive Bearbeitung erfordert daher eine entsprechend differenzierte politische Strategie, für deren Umsetzung ein breiter Fächer an Instrumenten zur Verfügung stehen muss. Mit der 2003 verabschiedeten Europäischen Sicherheitsstrategie (ESS) verfügt die Union über ein erstes strategisches Grundlagendokument, das die wesentlichen sicherheitspolitischen Herausforderungen und Bedrohungen definiert. Darin betont sie den komplexen Charakter künftiger Krisen und die Notwendigkeit eines je nach Lage anzupassenden Instrumentenmixes. ${ }^{8}$ Zudem hat sie sich seit 1999 im Rahmen der ESVP militärische und zivile Mittel zugelegt und deren weiteren Ausbau bis 2010 (Military Headline Goal) ${ }^{9}$ beziehungsweise bis 2008 (Civilian Headline Goal) ${ }^{10}$ beschlossen. Schließlich verfügt sie bereits innerhalb der ersten Säule über gemeinschaftliche Instrumente, die zur akuten ${ }^{11}$ und zur strukturellen Krisenbearbeitung ${ }^{12}$ eingesetzt werden können. Die noch zu lösende Aufgabe besteht darin, die beteiligten EU-Akteure und die vielfältigen Instrumente so miteinander zu verzahnen, dass sie einer effektiven Krisenbearbeitung im Rahmen einer umfassenden Strategie des Krisenmanagements dienlich sind. Da die Union selbst ein komplexer Akteur ist, dessen sicherheitspolitische Handlungsfähigkeit von den Mitgliedstaaten abhängt und dessen Instrumente unterschiedlichen institutionellen Verfahren und damit auch Entscheidungsprozessen unterliegen, stellt die Schaffung einer kohärenten Struktur für die effektive Krisenbearbeitung eine anhaltende Herausforderung dar.

8 Vgl. European Security Strategy.

9 Das Headline Goal 2010 wurde am 17./18. Juni 2004 vom Europäischen Rat beschlossen. Es ist eine Anpassung und Erweiterung des Helsinki-Planziels von 1999. Seine Umsetzung soll die Union in die Lage versetzen, die erweiterten Petersbergaufgaben durchführen zu können. Diese umfassen seitdem neben humanitären Aufgaben und Rettungsaktionen, friedenswahrenden Aufgaben sowie Kampfeinsätzen bei der Krisenbewältigung, einschließlich Maßnahmen zur Herbeiführung des Friedens, auch gemeinsame Abrüstungsoperationen, die Unterstützung von Drittländern im Kampf gegen den Terrorismus und die Reform des Sicherheitssektors. Bis 2010 sollen die Mitgliedstaaten in der Lage sein, innerhalb von fünf Tagen über eine Militäroperation politisch zu entscheiden und sie nicht später als zehn Tage nach der Entscheidung zu beginnen. Dementsprechende Fähigkeiten sollen entwickelt werden. Vgl. Headline Goal 2010, in: EU security and defence. Core documents 2004, Chaillot Papers 75/2005, S. 111-116.

10 Der Europäische Rat verabschiedete am 16./17. Dezember 2004 das zivile Headline Goal 2008. Zusätzlich zu den im Jahr 2000 festgelegten prioritären Bereichen für die nichtmilitärischen Krisenmanagementfähigkeiten soll die Union befähigt werden, verschiedene Arten von Monitoring-Missionen durchzuführen, Beiträge zu leisten zur Sicherheitssektorreform und zur Entwaffnung, Demobilisierung und Wiedereingliederung von Kämpfern, lokale Institutionen durch Training und Monitoring oder durch Übernahme von Exekutivfunktionen (substitution mission) zu stärken oder aufzubauen. Es ist vorgesehen bis zu zehn zivile Operationen gleichzeitig durchführen zu können, darunter eine großangelegte substitution mission in nicht befriedeter Umgebung. Auf der Basis von Modulen sollen Krisenreaktionsteams aufgebaut werden, die in fünf bis zehn Tagen nach der politischen Entscheidung stationierbar sind. Vgl. Civilian Headline Goal 2008, in: EU security and defence. Core documents 2004, S. 359-363.

11 Zum Beispiel das Amt für humanitäre Hilfe der EG (ECHO) und der Krisenreaktionsmechanismus (RRM).

12 Zum Beispiel Entwicklungshilfe und Außenwirtschaftpolitik. 


\section{Zur Architektur des zivil-militärischen Krisenmanagements}

Mit dem Amsterdamer Vertrag und dem Vertrag von Nizza schuf die Europäische Union die vertraglichen Grundlagen für die Durchführung von Krisenmanagementoperationen. Der EUV (Amsterdam) enthält im Wesentlichen drei Neuerungen. Erstens wurde das Amt des Hohen Vertreters für die GASP geschaffen, das 1999 Javier Solana übertragen wurde. Er ist zugleich Generalsekretär des Rates. Der Hohe Vertreter soll zur Formulierung, Vorbereitung und Umsetzung der GASP im Namen des Rates beitragen und der bislang disparaten Außenpolitik Stimme und Gesicht verleihen. Zweitens wurde ihm mit der Policy Unit ein Stab zur Seite gestellt, der mittlerweile circa 45 Mitarbeiter umfasst. Drittens wurden die so genannten Petersbergaufgaben in den EUV übernommen. Damit wurde Krisenbewältigung unter Einsatz militärischer Mittel zu einem Aufgabenbereich der Union.

Der EUV (Nizza) schuf mit dem Politischen und Sicherheitspolitischen Komitee (PSK) ein Kernstück des Krisenmanagements. Es setzt sich aus je einem in der jeweiligen Ständigen Vertretung in Brüssel installierten Vertreter der Mitgliedstaaten und einem Vertreter der Kommission zusammen und tagt in der Regel zwei Mal wöchentlich. ${ }^{13}$ Den Vorsitz übernimmt entweder das Land, das die Ratspräsidentschaft innehat oder der Hohe Vertreter. Das PSK soll die internationale Lage verfolgen, dem Rat Stellungnahmen zur GASP/ESVP vorlegen - etwa strategische Optionen - und die Durchführung vereinbarter Politiken überwachen. Zudem nimmt es unter der Verantwortung des Rates die politische Kontrolle und strategische Leitung von Operationen zur Krisenbewältigung wahr. Es fungiert also als zentrale Anlauf-, Clearing- und Durchführungsstelle für die zivile und militärische Krisenbewältigung im Rahmen der ESVP. Das PSK muss sich mit dem Ausschuss der Ständigen Vertreter (COREPER) abstimmen. Auch wenn dieser keine Kompetenzen im militärischen Bereich hat, so ist er doch gemäß Art. 207 EGV für die Vorbereitung und Ausführung der vom Rat beschlossenen Mandate zuständig. Folglich müssen alle Beschlüsse des PSK dem COREPER vorgelegt werden (siehe Abbildung 1).

Der Militärausschuss (EUMC) wurde mit Ratsbeschluss vom 22. Januar 2001 geschaffen. Der aus den Generalstabschefs der Mitgliedstaaten beziehungsweise ihren Vertretern bestehende EUMC ist das oberste militärische Organ in den neuen politisch-militärischen Strukturen des Rates. ${ }^{14}$ Er berät das PSK in allen militärischen Fragen und übernimmt die militärische Führung bei allen entsprechenden Aktivitäten. Der EUMC ist vor allem verantwortlich für die Beurteilung der Krisenentwicklung, die Erarbeitung der militärischen Aspekte des Krisenmanagementkonzepts und die militärische Durchführung von Operationen. Ihm arbeitet der Militärstab (EUMS) zu. Er hat den Auftrag, sich mit der Frühwarnung, der Lagebeurteilung und der strategischen Planung im Hinblick auf die Ausführung der Petersbergaufgaben, einschließlich der Bestimmung der jeweiligen europäischen nationalen und multinationalen Streitkräfte, zu befassen und Beschlüsse gemäß den Vorgaben des Militärausschusses durchzuführen. Der EUMS ist ein aus 147 Mitarbeitern bestehender multinationaler Stab, der bislang über keine strategisch-operativen Führungsfähigkeiten verfügt. Diese Aufgabe übernimmt entweder die NATO gemäß dem Berlin-Plus-Abkommen ${ }^{15}$ oder das Hauptquartier (HQ) einer so genannten framework nation ${ }^{16}$. Allerdings wird gegenwär-

13 Dienstags und freitags, gegebenenfalls auch öfter.

14 Falls erforderlich nimmt auch der Befehlshaber der laufenden Operation teil.

15 Dieses regelt den gesicherten Zugang der Union zu den operativen Planungsmöglichkeiten der NATO und die Nutzung von NATO-Kommandostrukturen bei EU-geführten Operationen.

16 Die framework nation ist für die Führung, Verwaltung und Logistik eines multinational besetzten nationalen Hauptquartiers zuständig. Gegenwärtig verfügen fünf Staaten über diese Führungsfähigkeit: Deutschland, Frankreich, Griechenland, Großbritannien und Italien. 
tig im EUMS eine Zivil-Militärische Zelle aufgebaut, die auch operative Planungs- und Führungsaufgaben übernehmen soll. ${ }^{17}$

\section{Abbildung 1: Zivile und militärische Akteure im EU-Krisenmanagement}

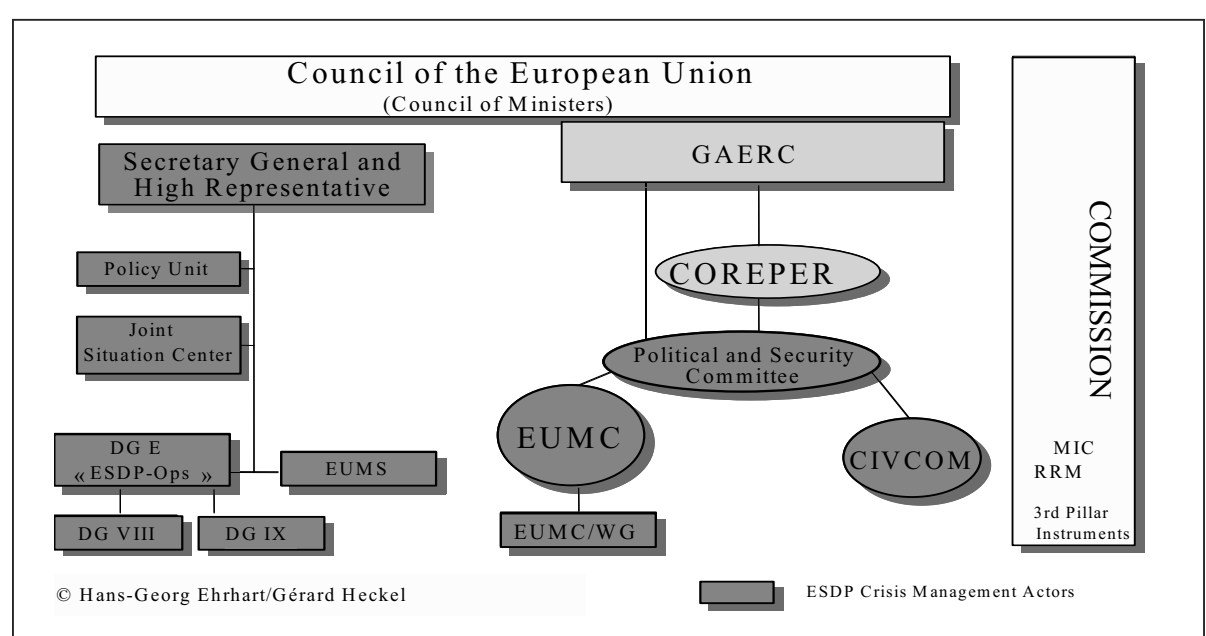

CIVCOM = Committee for Civilian Aspects of Crisis Management COREPER $=$ Comité des représentents permanents

$D G=$ Direction Général

$E U M C=E U$ Military Committee

EUMS $=$ EU Military Staff
GAERC = General Affairs and External Relations Council $M I C=$ Monitoring and Information Centre RRM = Rapid Reaction Mechanism $W G=$ Working Group

Für das zivile Krisenmanagement wurde ein Ausschuss gegründet (CIVCOM), der aus Vertretern aller Mitgliedstaaten und der Kommission besteht. Er soll dem PSK entsprechende Informationen liefern, Empfehlungen formulieren und Vorschläge unterbreiten sowie die Kohärenz der zivilen Aspekte des Krisenmanagements zwischen den Pfeilern gewährleisten. Der Ausschuss wird bezüglich seines Zuständigkeitsbereichs von den Beamten der Generaldirektion (GD) E für Auswärtige und Politisch-Militärische Angelegenheiten des Ratssekretariats unterstützt. Ihr unterstehen die zur Zeit gekaderte GD E VII (ESVP), die 30 Personen umfassende GD E VIII (Verteidigung) und die 40 Mitarbeiter zählende GD IX (Ziviles Krisenmanagement), worunter sich auch die von den Mitgliedstaaten abgeordneten Experten für Polizeimissionen befinden. Die GD E IX dient zugleich als Hauptquartier für zivile ESVP-Operationen. In ihr ist auch der 2000 eingerichtete pfeilerübergreifende zivile Krisenmechanismus untergebracht. Dabei handelt es sich um eine Datenbank, in der die von den Mitgliedstaaten potenziell zur Verfügung gestellten zivilen Mittel und der Grad ihrer Einsatzbereitschaft gespeichert sind. In der GD E VIII ist ferner das Satellitenzentrum der Europäischen Union angesiedelt. Schließlich verfügt der Hohe Vertreter über ein zivil-militärisches Lagezentrum.

Bei der Kommission sind weitere Instrumente für das akute Krisenmanagement der Union installiert. So ermöglicht der Rapid Reaction Mechanism (RRM) die kurzfristige Finanzierung von zivilen Maßnahmen zur Krisenbewältigung. Im Bereich des Zivilschutzes gibt es einen

17 Vgl. dazu weiter unten. Mit der Zivil-Militärischen Zelle hat die EU zwei Jahre nach dem viel kritisierten ,Pralinengipfel ‘ vom April 2003, auf dem Belgien, Deutschland, Frankreich und Luxemburg die Aufstellung eines europäischen Hauptquartiers ankündigten, Schritte eingeleitet, die genau in diese Richtung führen können. 
Mechanismus, der die Reaktionsfähigkeit auf diesem Gebiet, dem seit dem 11. September 2001 besondere Bedeutung beigemessen wird, erhöhen soll. Dieser umfasst das Monitoring and Informationen Center (MIC), das eine Datenbank über die verfügbaren zivilen Mittel der Mitgliedstaaten pflegt. Parallel existiert im EUMS eine Datenbank über militärische Mittel und Fähigkeiten zum Schutz der Zivilbevölkerung gegen die Folgen von Terroranschlägen.

\section{Herausforderung für die zivil-militärische Koordinierung}

Die oben erwähnten Akteure, Mittel und Fähigkeiten für ein umfassendes Krisenmanagement erfordern ein hohes Maß an Koordinierung. Die zentrale Herausforderung besteht darin, dass die relevanten Akteure die notwendigen zivilen und militärischen Mittel in koordinierter Weise und der jeweiligen Lage angepasst über den gesamten Prozess der Konfliktbearbeitung effektiv zum Einsatz bringen. Idealtypisch wird dieser Prozess in Abbildung 2 beschrieben.

\section{Abbildung 2: Zivile und militärische Anteile der Konfliktbearbeitung}

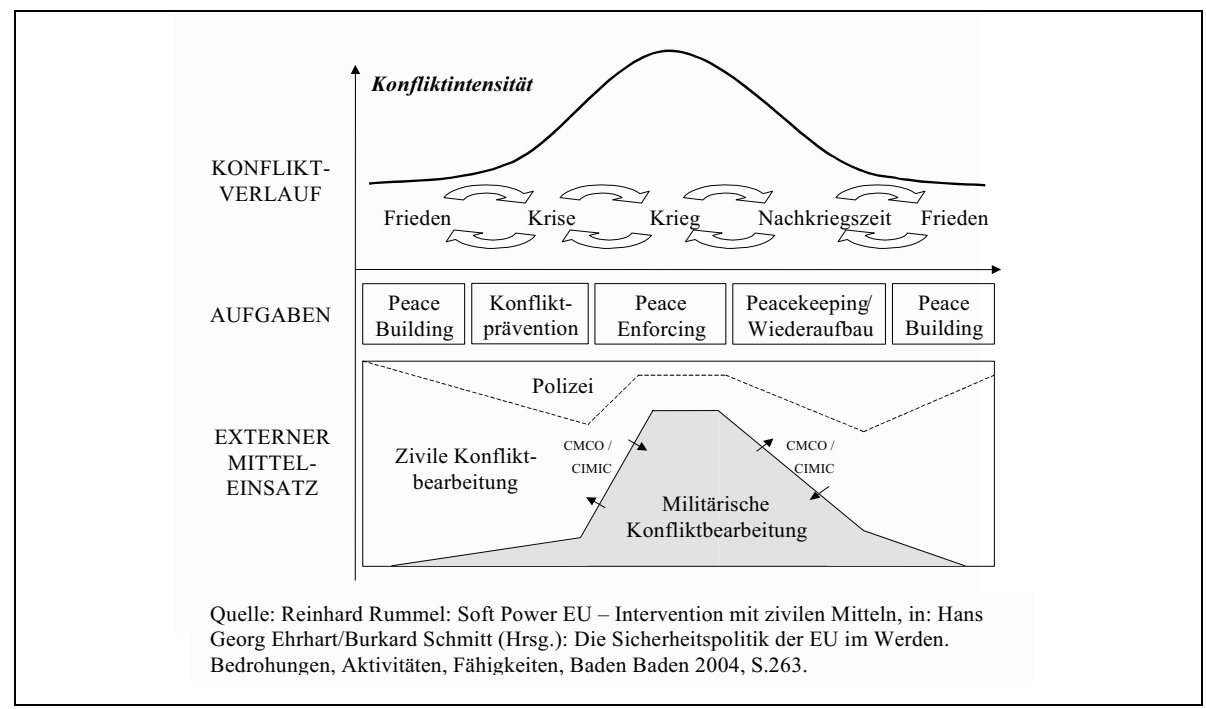

In der Union wird aus gutem Grund von der Notwendigkeit einer Kultur der Koordinierung gesprochen, um ein ausreichendes Maß an Kohärenz zu gewährleisten. Diese soll durch die effektive zivil-militärische Koordinierung (CMCO) aller relevanten EU-Akteure, die bei der Planung und Umsetzung des Krisenmanagements beteiligt sind, erreicht werden. Bislang gibt es noch kein ausgereiftes CMCO-Konzept. Erste Überlegungen wurden 2001 unter der schwedischen Präsidentschaft angestellt. Nach heutigem Verständnis umfasst CMCO in erster Linie die EU-interne Koordinierung des Krisenmanagements, denn diese ist die Voraussetzung für das kohärente Auftreten als externer Akteur. Es beinhaltet aber auch die Aufgabe, sich mit anderen Akteuren im Krisengebiet abzustimmen, etwa mit Nichtregierungsorganisationen, internationalen und nationalen Organisationen oder lokalen Akteuren. ${ }^{18}$ Die interne Koordinierung hat einen effektiven Mitteleinsatz im Rahmen eines kohärenten Gesamtansatzes zum Ziel, die externe Koordinierung dient der optimalen Errei-

18 Vgl. Council of the European Union, Civil-Military Coordination (CMCO), 14457/03, Brüssel 2003. 
chung der politischen Ziele einer Krisenoperation. CMCO ist vom bereits fortgeschritteneren Konzept der zivil-militärischen Zusammenarbeit (CIMIC) zu unterscheiden. ${ }^{19}$ Demnach ist CIMIC auf die Beziehungen zwischen den militärischen Komponenten und den zivilen, externen Akteuren gerichtet und umfasst die drei Aufgaben zivil-militärische Verbindung, Unterstützung des militärischen Auftrags und Unterstützungsleistungen für das zivile Umfeld. Im Gegensatz zur NATO verfügt die Union aber über zivile Mittel und Fähigkeiten zur Krisenbearbeitung, so dass sie ein anderes Verständnis von ,zivilen Akteuren“ und deren Rolle hat. Dieses findet seinen Ausdruck darin, dass CIMIC in der Union bereits auf der politisch-strategischen Ebene Teil des Krisenmanagementprozesses ist. ${ }^{20}$ Insofern ist CIMIC als Teil von $\mathrm{CMCO}$ zu verstehen. Letztlich geht es um die Koordinierung aller EU-Aktivitäten, wie Abbildung 3 schematisch verdeutlicht.

\section{Abbildung 3: Zivil-militärische Koordinierung}

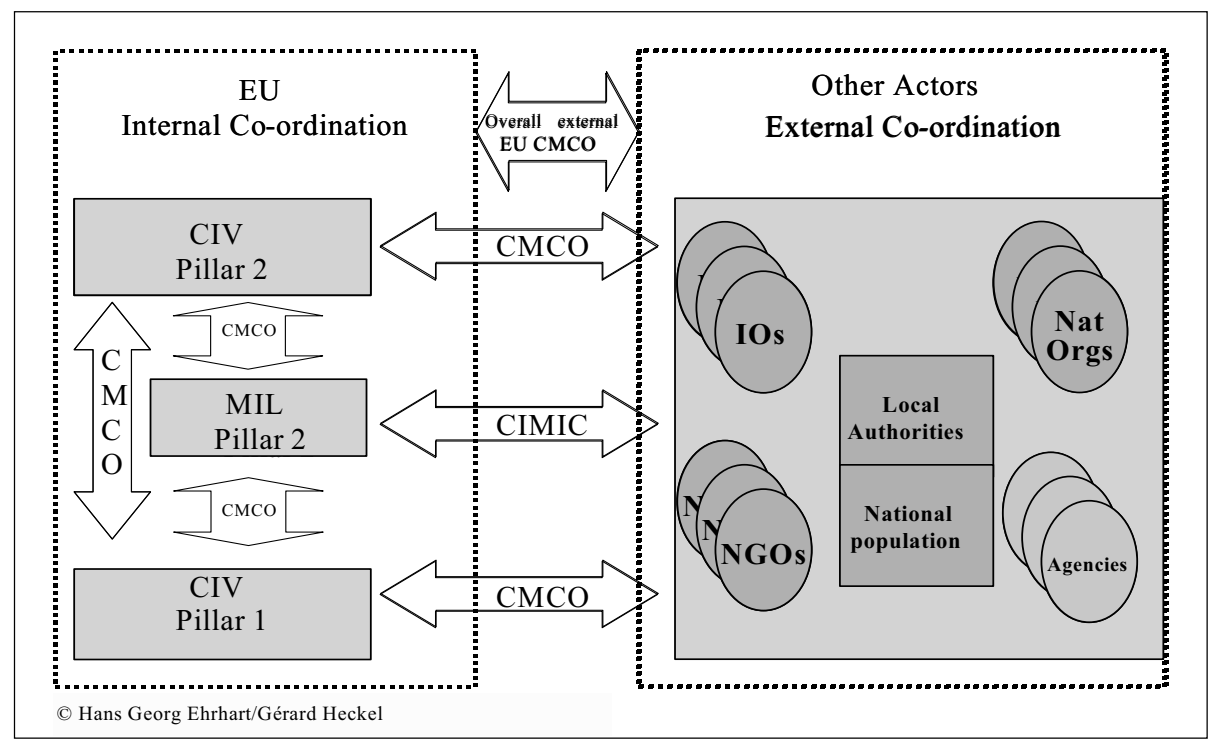

CIMIC $=$ Civil-Military Cooperation

CMCO = Civil-Military Coordination

$C I V=$ Civilian Instruments

IOs = International Organisations
Nat Orgs = National Organisations

NGOs = Non-governmental Organisations

$M I L=$ Military Instruments

\section{Krisenmanagementkonzept und -prozess}

Die Implementierung einer umfassenden Krisenbearbeitung erfordert ein gemeinsames übergreifendes Konzept und - angesichts der komplexen Struktur der Europäischen Union flexible Mechanismen und Verfahren für die Koordinierung der Akteure und Instrumente. Die primäre Aufgabe dieses Konzepts besteht also darin, die interne Koordinierung und Vernetzung auf den verschiedenen Ebenen - politisch-strategisch, militärisch-strategisch, militärisch-operationell und taktisch - zu gewährleisten. Abbildung 4 verdeutlicht zum ei-

19 Vgl. Council of the European Union, Civil-Military Cooperation (CIMIC) concept for EU-led Crisis Management Operations, 7106/02, Brüssel 2002. Vgl. auch die Dokumentation von Daniel Wenig: Zivil-militärische Zusammenarbeit in der Europäischen Union, Aktuelle SWP-Dokumentation, Reihe D, Nr. 46, Juli 2004.

20 Das kann zur Folge haben, dass es bei der Übernahme von NATO-Operationen durch die EU zu Problemen kommt. 
nen, dass es eine klare Trennung zwischen den zivilen Mitteln der Gemeinschaft und denjenigen des zweiten Pfeilers gibt. Zum anderen zeigt sie, dass es mehrere Entscheidungsstränge mit unterschiedlichen Zuständigkeiten und Verfahren gibt.

So übernimmt im Fall eines Kriseneinsatzes der EUMC die militärische Führung und erteilt dem Befehlshaber der Operation entsprechende Weisungen. Die Kommission benennt einen Head of Commission Office, der dem PSK direkt berichtet. Für die polizeilichen und zivilen Aktivitäten werden ein Police Head of Mission (PolHoM) und ein Head of Mission (HoM) ernannt.

\section{Abbildung 4: Akteure, Ebenen und Vernetzung im Krisenmanagementprozess}

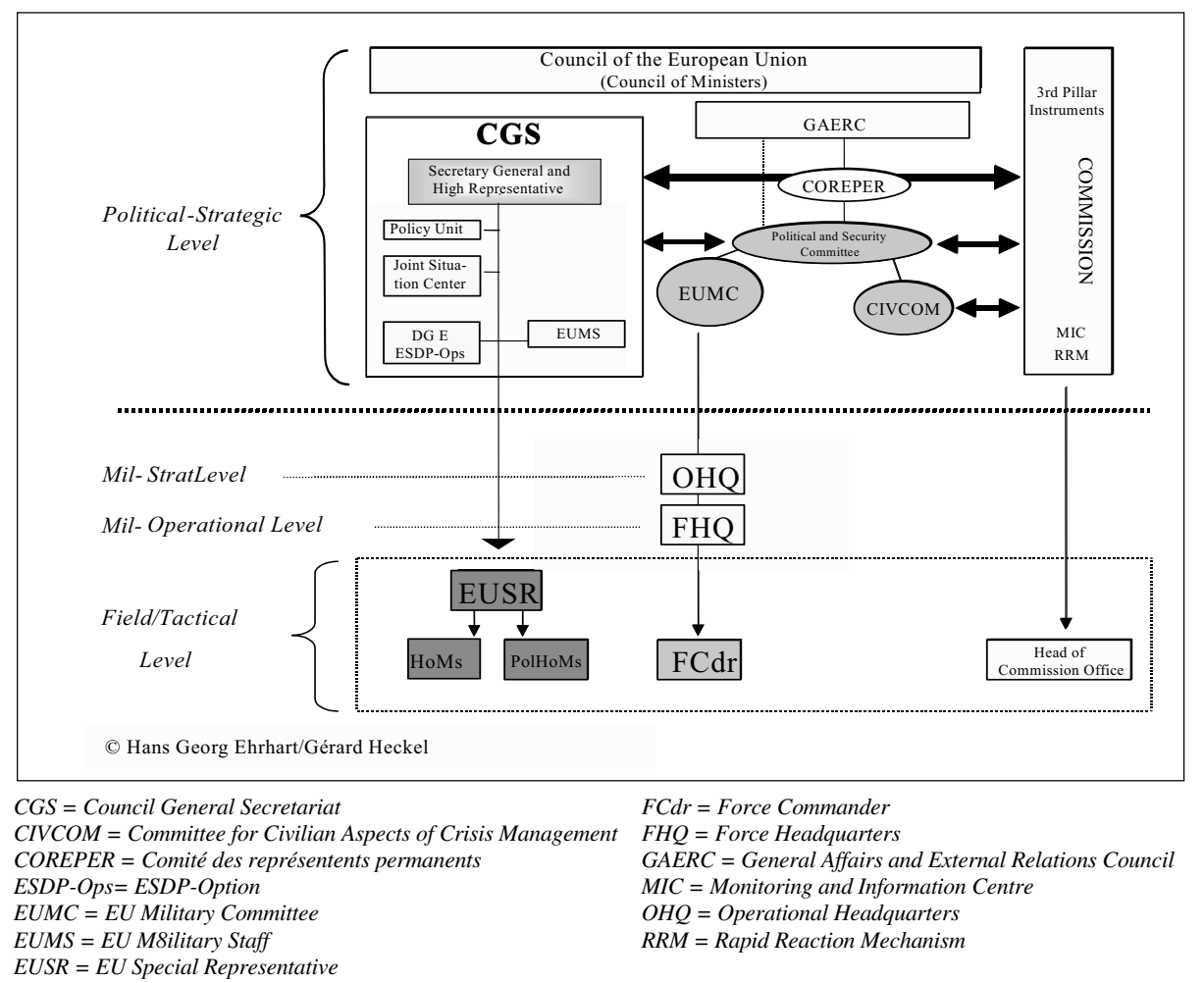

Um das Koordinierungsproblem in den Griff zu bekommen, erstellt die Union ein vom Rat abzusegnendes Krisenmanagementkonzept (Crisis Management Concept, CMC), das die Grundlage für die zu erarbeitenden verschiedenen strategischen Optionen ist. Diese können eine zivile (Civil Strategic Option, CSO), eine polizeiliche (Police Strategic Option, PSO), eine militärische (Military Strategic Option, MSO) und eine Option der Kommission (EC Measures) umfassen. Nach der Genehmigung durch das PSK werden daraus die verschiedenen Operationskonzepte (Concept of Operation, CONOPS) erarbeitet, die wiederum - nach Genehmigung durch den Rat - als Grundlage für die Erarbeitung der Operationspläne (Operation Plan, OPLAN) dienen.

Wichtigstes Koordinierungsinstrument ist das Krisenmanagementkonzept. Dabei handelt es sich um ein ausführliches und flexibles Dokument, das den übergreifenden Ansatz der Ope- 
ration detailliert beschreibt. Ausgehend von einer Lage- und Risikoanalyse sowie der Beschreibung der Interessen und Ziele benennt es die Einsatzoptionen und die verschiedenen, zum Einsatz vorgesehenen Instrumente, beschreibt die Modalitäten ihrer Koordinierung und regelt die Abstimmung zwischen gemeinschaftlichen und zwischenstaatlichen Instrumenten. Zudem werden die Maßnahmen nach den verschiedenen Zeithorizonten (kurz-, mittel-, langfristig) differenziert sowie die Koordinierung mit externen Akteuren eingeplant. Das CMC ist Teil eines Krisenmanagementverfahrens, für welches die Union detaillierte Verfahrensvorschläge (einschließlich für Krisen von höchster Komplexität) in Form eines Flussdiagramms entwickelt hat. Dieses unterscheidet schematisch zwischen sechs Phasen: Routinephase, Krisenbeginn, Annahme des CMC und Entwicklung der strategischen Optionen, formale Entscheidung für eine Operation und Entwicklung der Planungsdokumente, Implementierung, Anpassung und Beendigung der EU-Operation. ${ }^{21}$ Das Dokument ist keine verpflichtende Vorgabe, sondern dient als Referenzrahmen für die Identifizierung der Verfahren für ein kohärentes und umfassendes Krisenmanagement. Angesichts neuer Anforderungen an die schnelle Reaktionsfähigkeit durch das Headline Goal 2010 muss das Konzept allerdings angepasst werden.

Die Koordinierung von Akteuren und Maßnahmen erfolgt im Laufe des Krisenmanagementprozesses auf verschiedenen Ebenen und in unterschiedlichen Formaten (siehe Abbildung 5). Auf der politisch-strategischen Ebene wird ein Crisis Response Coordination Team (CRCT) eingesetzt. Dieses Ad-hoc-Team besteht aus Vertretern aller am Krisenmanagement beteiligten Akteure. Es tritt auf der Ebene der Generaldirektoren zusammen und variiert je nach Zuschnitt der geplanten Operation. Die wichtigste Aufgabe des CRCT ist es, den Entwurf des Krisenmanagementkonzepts zu erarbeiten. In späteren Phasen sollen regelmäBige Besprechungen dazu beitragen, die Lage zu verfolgen und gegebenenfalls Anpassungsmaßnahmen vorzuschlagen. ${ }^{22}$

Daneben wird im Ratssekretariat ein Crisis Action Team (CAT) eingerichtet. Die Aufgabe dieser Ad-hoc-Gruppe ist es vor allem, die militärische strategische Option zu verfassen. Zudem ist das CAT Ansprechpartner für das militärstrategische Hauptquartier. Die Koordinierung mit der GD E VIII und IX erfolgt durch inoffizielle Informations- und Abstimmungstreffen. Die Koordinierung zwischen der militärstrategischen Ebene - also entweder dem Hauptquartier der framework nation oder demjenigen der NATO - und den zivilen Akteuren der Europäischen Union soll durch die Abstellung von Verbindungspersonen durch alle beteiligten Akteure an das strategische Hauptquartier und die Entsendung eines Vertreters des $\mathrm{OHQ}$ an das CAT gewährleistet werden. Auf der operativ-taktischen Ebene soll der EUSonderbeauftragte (EUSR) als zentrale Koordinierungsstelle agieren. Er leitet die konkreten Koordinierungsbemühungen vor Ort durch eine Koordinierungsgruppe, an der Vertreter aller EU-Institutionen teilnehmen können.

21 Council of the European Union, Suggestions for procedures for coherent, comprehensive EU crisis management, 11127/03, Brussels 2003.

22 Allerdings gibt es noch immer interne Diskussionen darüber, ob das CRCT oder das Task-Force-Modell, wie es zur Zeit für den Balkan und Darfur praktiziert wird, das geeignete Koordinierungsinstrument ist. Für die Operation Althea spielte das CRCT eine geringe Rolle. Gleichwohl steht es als einziges hochrangiges Koordinierungsinstrument zur Verfügung, während die Task-Forces eher für die Koordinierung zwischen den Ressorts (desk-level) zuständig sind. 
Abbildung 5: Koordinierungsteam für Krisenreaktion und Krisenreaktionsteam

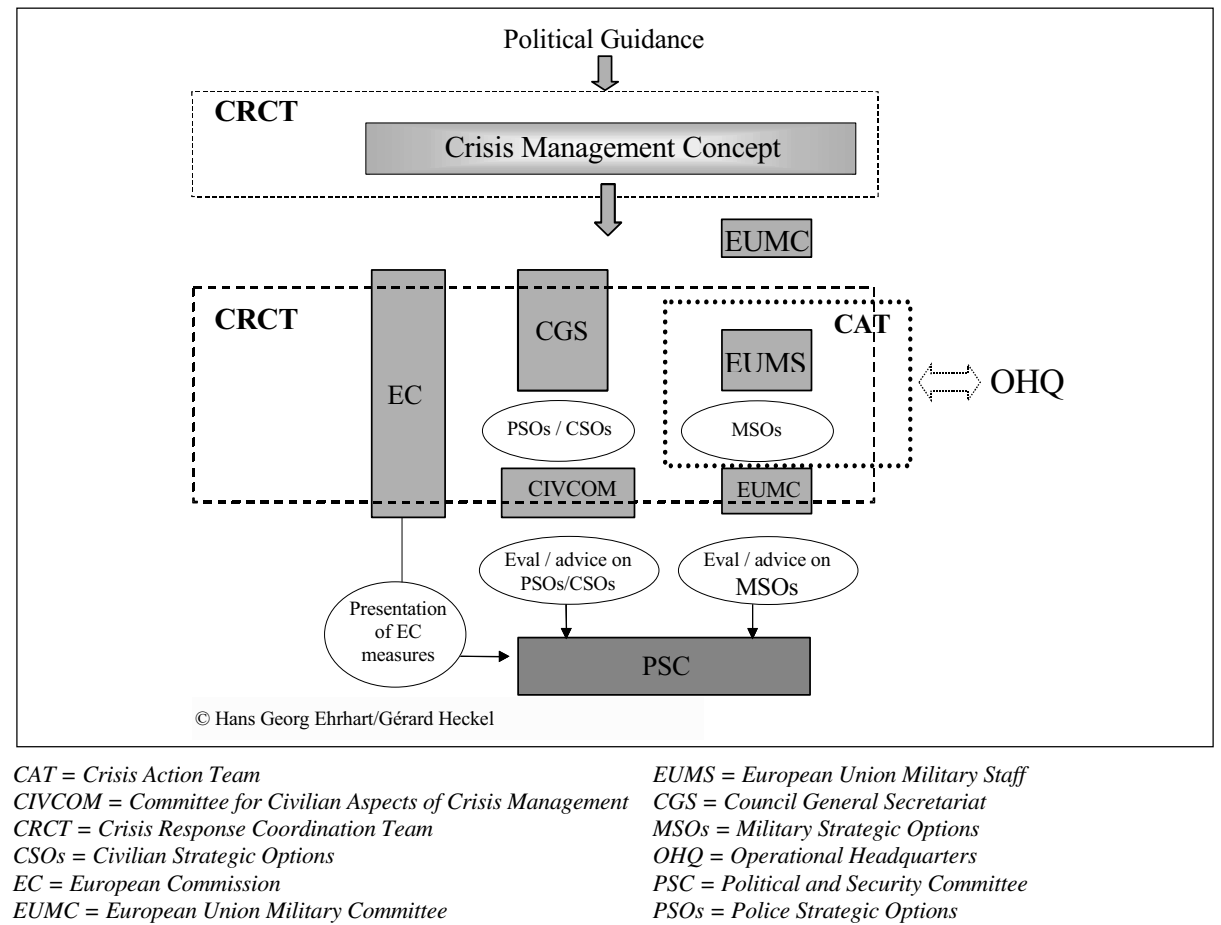

\section{Probleme der zivil-militärischen Koordination}

Die oben erfolgte Darstellung der zivil-militärischen Koordinierung im Rahmen der Planung und Umsetzung von Krisenmanagementoperationen lässt bereits erahnen, dass es sich dabei nicht um einen reibungslosen Prozess handelt. Überspitzt ausgedrückt könnte man sagen, dass alle koordinieren wollen, aber keiner koordiniert werden will. Auf Grund der unterschiedlichen Zuständigkeiten und der Governance-Strukturen der Union ist eine direkte Anordnung beziehungsweise Verpflichtung der verschiedenen Akteure zur CMCO nicht möglich. Dementsprechend wird in den einschlägigen Dokumenten lediglich empfohlen und nahegelegt. Ob und wie sich die Akteure daran halten, ist eine andere Frage. Hinzu kommen Ressortegoismen und die Furcht vor Kompetenzverlust beziehungsweise Dominierung durch andere. Probleme mit CMCO können auf drei Ebenen identifiziert werden: interinstitutionell, intrainstitutionell und vor Ort.

Zum Ersten ist die Rivalität zwischen der Kommission und den Akteuren des zweiten Pfeilers vor allem strukturell und kulturell bedingt. Beide betreiben Außenpolitik, allerdings auf verschiedenen vertraglichen Grundlagen, nach unterschiedlichen Verfahren und mit anderen Methoden. Auch vom politisch-kulturellen Selbstverständnis her handelt es sich um zwei verschiedene Welten oder ,Stämme', mit dem Ergebnis, dass ,the EU is pursuing a bifurcated foreign policy: politics is dealt with in the inter-governmental second pillar as a declaratory and pennyless exercise; substantive and funded external politics belong to the first pillar and are implemented by the Commission“. ${ }^{23}$ Im Grundsatz sind sich alle Beteiligten

23 Gilles Andréani/Christoph Bertram/Charles Grant: Europe's Military Revolution, London 2001, S. 43. 
einig, dass komplexes Krisenmanagement ein hohes Maß an interinstitutioneller Koordinierung notwendig macht. Die Frage ist aber, wo die Grenzen der Koordinierungsbereitschaft und -fähigkeit liegen und wie diese überwunden werden können. So ist der Einfluss der Kommission auf die ESVP eher gering. Ihre Aktivitäten sind zum größten Teil mittel- und langfristig auf strukturelle Krisenprävention ausgerichtet und die Durchführung erfolgt in eigener Zuständigkeit und oftmals über Nichtregierungsorganisationen, die, wenn die Haushaltsmittel einmal bewilligt worden sind, weitgehend autonom handeln. Das gilt auch für die für humanitäre Hilfe zuständige Agentur ECHO, die sich als unpolitischer Akteur versteht und nicht als Instrument der Krisenbewältigung. ${ }^{24}$ Die Kommission sieht sich im Bereich ziviler Krisenbearbeitung in direkter Konkurrenz zu den zivilen Akteuren des zweiten Pfeilers, insbesondere wenn es um den Zivilschutz geht. Sie lehnt bislang auch eine Beteiligung am CAT ab, weil sie Kompetenzverluste befürchtet. In der intergouvernementalen Struktur der GASP wird wiederum der Kommission mangelnde Transparenz nachgesagt. Zudem wird jede Form von Vergemeinschaftung abgelehnt. Immerhin verfügt die Kommission über viel mehr Geld und außenpolitisches Personal. ${ }^{25}$ Darum ist auch der Rat äußerst zurückhaltend, wenn es um die Einbindung der Kommission in die ESVP geht.

Zum Zweiten existieren auch innerhalb des zweiten Pfeilers Reibungspunkte vor allem zwischen zivilen und militärischen Akteuren. Beide Strukturen wurden ja geschaffen, um die jeweiligen Aktivitäten im Rahmen des Krisenmanagements zu planen und umzusetzen. Allerdings ist die personelle Ausstattung von Beginn an durch eine Schieflage zu Gunsten der militärischen Seite gekennzeichnet. So arbeiten im EUMS 147 Offiziere (die gegebenenfalls noch durch die Führungsstäbe der framework nation oder der NATO unterstützt werden), während für die zivile Krisenbearbeitung, die ja gemäß EU-Rhetorik eigentlich im Mittelpunkt stehen soll, lediglich 70 Ratsbeamte und abgeordnete nationale Experten zur Verfügung stehen. ${ }^{26}$ Die Unterbesetzung der GD E IX führt zum Beispiel dazu, dass das auf allen Ebenen vorgesehene Verbindungspersonal de facto nicht zur Verfügung gestellt werden kann - mit entsprechenden Auswirkungen auf die CMCO. Des Weiteren scheint die Koordinierung zwischen den zivilen und militärischen Fachreferaten nicht optimal zu funktionieren. Dafür gibt es verschiedene Ursachen: Die Zivilisten der GD E sind es ebenso wenig gewöhnt mit einer militärischen Institution zu arbeiten wie umgekehrt. Die politischen Kulturen sind mithin noch zu unterschiedlich. Hinzu kommt, dass beide Bereiche meist durch eine geschlossene Tür getrennt sind. Dadurch wird die inoffizielle Koordinierung erschwert. Eine Formalisierung der Beziehungen ist bislang auch nicht möglich gewesen, weil dann die sensible Frage der Hierarchisierung aufgeworfen werden könnte. Weder wollen sich die Militärs von den meist noch jungen Zivilisten dominieren lassen, noch wollen diese dem Militär eine Vorrangstellung zubilligen. Die unzureichende Formalisierung trägt also zu Koordinierungsproblemen bei. Ein weiteres Problem besteht darin, dass das militärische Planungsverfahren im Hauptquartier stattfindet, also gegenwärtig entweder im NATOHauptquartier SHAPE oder im Hauptquartier der zuständigen framework nation. Der vorgesehene permanente Austausch von Verbindungselementen ist zurzeit aber auf Grund personeller Knappheit kaum zu leisten. Erschwerend kommt hinzu, dass die im OHQ mit der Erstellung des Operationskonzepts beauftragten Soldaten keine Erfahrung in der Operationsplanung für ein umfassendes Krisenmanagement haben. Während die Offiziere also in ihrem OHQ planen und dort auch den Operationsplan erstellen, werden die zivilen

24 Daher lehnte ECHO bislang die Teilnahme an der Erstellung eines Krisenmanagementkonzepts ab.

25 Der Kommissarin für Außenbeziehungen unterstehen über 6.400 Beamte. Ihr Etat umfasst mehr als sieben Mrd. Euro. Vgl. Süddeutsche Zeitung, 21./22.5.2005.

26 Insgesamt arbeiten im Bereich der ESVP gegenwärtig also 286 Personen (Stand 11.5.2005). 
Konzepte und Pläne im Ratssekretariat beziehungsweise von der Kommission entworfen und abgestimmt.

Zum Dritten ist zu fragen, warum die zivilen Operationsleiter erst später in die Operationsplanung eingebunden werden als der militärische Operationsleiter. Die Existenz paralleler Befehlsketten erleichtert natürlich nicht die Koordinierung vor Ort. Welche Instrumente sind in einer konkreten Situation einzusetzen? Wer ist wofür zuständig? Wer hat die Entscheidungskompetenz? Wer soll das Ganze koordinieren? Die erste Militäroperation der Union (Operation Concordia) hat die Bedeutung eines starken Koordinators bestätigt. Die Stellung des EU-Sonderbeauftragten war damals zu schwach. Der EUSR muss einerseits mehr sein als ein machtloser politischer Berater und Repräsentant, kann andererseits aber auch nicht Teil der militärischen Befehlskette sein. Gleichwohl bedarf er erweiterter Kompetenzen im Bereich der Vor-Ort-Koordinierung. Andere während der Operation aufgetretene Schwachstellen betrafen neben der Umsetzung des Berlin-Plus-Abkommens, Führungsfragen, Aufklärung und Kommunikationssicherheit auch das Krisenmanagementverfahren und CMCO. Ein Grund dafür ist in dem Umstand zu sehen, dass Concordia verspätet und unter hohem Zeitdruck gestartet werden musste. Eine andere Ursache liegt darin, dass es sich dabei um die erste Militäroperation handelte, die operative Lernkurve also bei Null begann. ${ }^{27}$

Mittlerweile hat die Europäische Union bereits Erfahrungen aus neun ESVP-Missionen: Die zivilen Polizeieinsätze in Bosnien (EUPM), Mazedonien (EUPOL Proxima) und in der Demokratischen Republik Kongo (EUPOL Kinshasa), wo seit dem 8. Juni 2005 auch die erste ESVP-Mission zur Reform des Sicherheitssektors läuft (EUSEC DR Congo), die rule of law-Missionen in Georgien (EUJUST THEMIS) und im Irak (EUJUST Lex), die (abgeschlossenen) Militäreinsätze in Mazedonien und in der Demokratischen Republik Kongo (Artemis) sowie die seit dem 2. Dezember 2004 laufende, erste umfangreiche Militäroperation in Bosnien-Herzegowina (ALTHEA). ALTHEA ist eine komplexe Operation, die höchste Ansprüche an die zivil-militärische Koordinierung stellt. Die Operation ist laut Mandat Teil eines kohärenten Ansatzes, indem sie die umfassende Politik der Union gegenüber Bosnien-Herzegowina stärkt und sich eng mit der übrigen EU-Präsenz im Lande koordiniert. Für die Gesamtkoordinierung ist der EU-Sonderbeauftragte verantwortlich. Die Militärkommandeure wiederum, will coordinate closely with the EUSR in $\mathrm{BiH}$ with a view to ensuring consistency of the EU military operation with the broader context of the EU activities in $\mathrm{BiH}^{\text {“ }}{ }^{28}$ Die Rolle des EUSR ist also politisch gestärkt worden. Wie sich das Ganze in der Praxis auswirkt, bleibt allerdings abzuwarten.

\section{Reformen und Reformmöglichkeiten}

Die operativen Aufgaben der ESVP nehmen seit 2003 stark zu. ${ }^{29}$ Verantwortlich dafür sind das dynamische internationale Umfeld und die damit verbundenen Herausforderungen. Sie setzen die GASP/ESVP unter hohen Reformdruck. Ob der Verfassungsvertrag der Europäischen Union (VVE) den laufenden Ratifizierungsprozess überstehen wird, ist zurzeit mehr als fraglich. Gleichwohl ist es bei aller Kritik, dass mehr wünschbar gewesen wäre, weitge-

27 Insgesamt ist die Operation Concordia positiv zu bewerten. Sie trug zur Stabilisierung der Lage in Mazedonien bei, ermöglichte den ersten Test des Berlin-Plus-Abkommens und unterzog die ESVP einem ersten Realitätstest, der zu wertvollen Erkenntnissen und Lehren insbesondere für die Umsetzung einer effektiveren CMCO führte. Vgl. Hans-Georg Ehrhart: Die EU als militärischer Akteur in Mazedonien: Lehren und Herausforderungen für die ESVP, in: Johannes Varwick (Hrsg.): Die Beziehungen zwischen NATO und EU. Partnerschaft, Konkurrenz, Rivalität?, Opladen 2005, S. 169-184.

28 EUFOR ALTHEA, in: EU security and defence. Core documents, Vol. V, Chaillot Paper 75/2005, S. 325.

29 Weitere zivile Einsätze stehen im Sudan und im Gazastreifen an oder werden erwogen. 
hend unstrittig, dass der VVE wichtige Reformen im Bereich der GASP/ESVP vorsieht. Drei davon - die Solidaritätsklausel, die Verteidigungsagentur und die Gefechtsverbände sind bereits vor Inkrafttreten politisch wirksam geworden. Zusammen mit anderen Reformschritten führen sie zu Anpassungen in den Bereichen Sicherheitszusagen, Fähigkeiten und institutionelle Reform/Entscheidungsprozess.

Was den ersten Aspekt betrifft, so sei zunächst Art. I-41 Abs. 7 VVE erwähnt. Er enthält eine Beistandsklausel, gemäß der im Falle eines bewaffneten Angriffs auf das Hoheitsgebiet eines Mitgliedstaates die anderen Mitglieder, ,nach Artikel 51 der Charta der Vereinten Nationen alle in ihrer Macht stehende Hilfe und Unterstützung leisten (müssen). " Die in Art. I43 VVE enthaltene Solidaritätsklausel bestimmt, dass die Union, ,alle ihr zur Verfügung stehenden Mittel, einschließlich der ihr von den Mitgliedstaaten bereitgestellten militärischen Mittel (mobilisiert)“, um die Mitgliedstaaten auf ihrem Hoheitsgebiet im Falle terroristischer Bedrohungen und Anschläge oder im Katastrophenfall zu schützen und solidarisch zu unterstützen. Gerade dieser Fall verlangt ein Höchstmaß an Koordination.

Hinsichtlich des zweiten Bereichs, der Fähigkeiten, seien drei Aspekte kurz angesprochen. Die Bestimmungen über die Europäische Verteidigungsagentur (Art. I-41 Abs. 3 VVE) verpflichten die Mitgliedstaaten zwar dazu, zivile und militärische Fähigkeiten für die ESVP zur Verfügung zu stellen. Doch wurde nur für den militärischen Bereich eine Agentur eingerichtet, die diesen Prozess koordinieren und unterstützen soll. Angesichts der wachsenden Relevanz ziviler Fähigkeiten im Krisenmanagement wäre es aber notwendig, einen ähnlichen Mechanismus auch für den zivilen Bereich einzurichten. Der Europäische Rat nahm im Dezember 2004 das Konzept für die Aufstellung von sehr schnell einsetzbaren Gefechtsverbänden an. ${ }^{30}$ Es sieht vor, dass bis 2007 dreizehn Verbände von je 1500 Soldaten für den Einsatz in schwierigem Umfeld aufgestellt werden, die auch - aber nicht ausschließlich - für Missionen der Vereinten Nationen zur Verfügung stehen sollen. Gerade bei einer humanitären Operation kommt es aber auf eine funktionierende interne und externe Koordinierung an, so dass es mit der Aufstellung der Gefechtsverbände allein nicht getan sein wird. Zudem muss der gesamte Entscheidungsprozess extrem beschleunigt werden, soll die Vorgabe von fünf Tagen bis zur Entscheidung über den Beginn einer Operation eingehalten werden. ${ }^{31}$ Was die interne Koordinierung betrifft, so soll die seit 2004 im EUMS im Aufbau befindliche Zivil-Militärische Zelle unter anderem die Koordinierung mit zivilen Operationen unterstützen. Weitere Aufgaben sind ,to enhance the EU's capacity for crisis management planning, to reinforce national HQ designated to conduct an EU autonomous operation (...) and to generate the capacity to plan and run an autonomous operation" ${ }^{32}$ Die Zivil-Militärische Zelle hat also die zweifache Aufgabe, politisch-strategische Planung zu leisten und Operationen durchzuführen. Dementsprechend besteht sie aus zwei Komponenten: Einer 16 Mitarbeiter umfassenden strategischen Planungseinheit (neun Militärs, sieben Zivilisten, davon zwei aus der Kommission) und einem permanenten Kern aus acht Offizieren, der das auf 89 Personen ausbaufähige Operationszentrum bildet. ${ }^{33}$ Die genaue Definition der Zuständigkeiten ist zur Zeit noch umstritten. Weder die ,Zivilisten' der GD E noch die der Kommission sind über eine mögliche Unterordnung unter den EUMS erfreut. Andererseits ist die Zelle bislang nur ein bloßer Annex des EUMS. Für die zivil-militärische Koordinierung wäre es

30 Vgl. auch Artikel 1 des 23. Protokolls über die Ständige Strukturierte Zusammenarbeit zum VVE.

31 Vgl. dazu General Affairs and External Relations Council (External Relations), 2660th Council Meeting, 23./ 24. 5. 2005, S. 8-9.

32 Background paper on civilian crisis management in ESDP, Brüssel 2005, S. 11

33 Der Personalumfang des EUMS wird also auf mindestens 171 und höchstens 252 zunehmen. Geleitet wird die Zivil-Militärische Zelle von einem Offizier, Stellvertreter ist ein Zivilist. 
effizienter, sie aus dem EUMS wieder herauszulösen und sie als übergreifende Koordinierungsinstanz der Arbeitsebene zu installieren, der EUMS und DG E zuarbeiten.

Was nun drittens schließlich die GASP-relevanten Aspekte der institutionellen Reform durch den VVE angeht, so muss im Hinblick auf die angestrebte Auflösung der Pfeilerstruktur konstatiert werden, dass diese in der GASP de facto weiterhin gilt. Die Einführung der verstärkten Zusammenarbeit in den gesamten Bereich der ESVP wird dadurch getrübt, dass sie nur einstimmig beschlossen werden kann (Art. III-419 Abs. 2 VVE). Die ständige strukturierte Zusammenarbeit im Verteidigungsbereich ${ }^{34}$ ermöglicht zweifellos flexiblere Entscheidungen, wird sie doch im Gegensatz zur verstärkten Zusammenarbeit durch eine Ratsentscheidung mit qualifizierter Mehrheit eingeführt. ${ }^{35}$ Allerdings können Entscheidungen innerhalb der ständigen strukturierten Zusammenarbeit nur im Konsens getroffen werden. Ein weiterer Kritikpunkt lautet, dass die Kommission weder bei der ständigen strukturierten Zusammenarbeit noch bei Operationen nach Art. I-40 Abs.1 VVE erwähnt wird. Das entspricht zwar dem Interesse der großen Mitgliedstaaten, die Kommission aus der ESVP möglichst herauszuhalten. Zugleich könnte aber diese Nichtbeachtung ,den Teilnehmern künftig eine potenziell sehr nützliche Unterstützung vorenthalten". 36

Drei weitere Reformvorhaben des VVE könnten zu einem Gewinn an Handlungsfähigkeit führen. Die zweieinhalbjährige Amtszeit des Präsidenten des Europäischen Rates dürfte auf jeden Fall für eine größere Kontinuität bei Festlegung der Prioritäten und der Außendarstellung sorgen. Als viel bedeutsamer könnte sich der in Art. I-28 VVE vorgesehene Außenminister der Union und der ihm zuarbeitende europäische diplomatische Dienst erweisen. Der Europäische Außenminister wird die GASP leiten und den Vorsitz im Rat „Auswärtige Angelegenheiten" führen. Er soll explizit für ein kohärentes auswärtiges Handeln Sorge tragen, ist also unter der Federführung des Rates für das zivil-militärische Krisenmanagement verantwortlich. Die Konstruktion des Doppelhutes - er ist zuständig für die GASP und zugleich Vizepräsident der Kommission und dort für die Koordinierung aller zu den äußeren Beziehungen der Gemeinschaft gehörenden Ressorts verantwortlich ${ }^{37}$ - soll die institutionelle Voraussetzung dafür schaffen. Wie dies in der Realität funktioniert, bleibt abzuwarten, denn die GASP wird weiterhin nach der intergouvernementalen Methode ablaufen, während der $\mathrm{Au}-$ ßenminister bei der Wahrnehmung der Zuständigkeiten der Kommission deren Arbeitsweise unterliegt, also der Gemeinschaftsmethode.

Um den Außenminister der Union bei der Vielfalt seiner Arbeiten zu unterstützen, sieht Art. III-296 Abs. 3 VVE die Einrichtung eines Europäischen Auswärtigen Dienstes vor. Dieser soll sich aus abgeordneten Mitarbeitern der nationalen diplomatischen Dienste sowie aus Beamten des Ratssekretariats (einschließlich EUMS) und der Kommission zusammensetzen. Strittig ist aber, wo und wie der neue Dienst institutionell verankert werden soll. Das Europäische Parlament befürwortet eine Einbindung in die Kommission, nationale Vertreter

34 Art. I-41 Abs. 6 VVE bestimmt, dass die Mitgliedstaaten, die anspruchsvollere Kriterien in Bezug auf die militärischen Fähigkeiten erfüllen und die im Hinblick auf Missionen mit höchsten Anforderungen untereinander weiter gehende Verpflichtungen eingegangen sind, eine ständige strukturierte Zusammenarbeit begründen. Vgl. dazu auch das 23. Protokoll des VVE.

35 Weitere Besonderheiten bei der ständigen strukturierten Zusammenarbeit bestehen darin, dass der VVE keine Mindestteilnehmerzahl vorsieht, dass nur die Teilnehmer über die Aufnahme neuer Mitglieder bestimmen, dass diese exklusiv tagen und selbst bestimmten Kriterien genügen müssen, deren Nichterfüllung gegebenenfalls Sanktionen nach sich ziehen kann.

36 Christian Deubner: Verstärkte Zusammenarbeit in der verfassten Europäischen Union, in: integration 2/2004, S. 274-284, hier: S. 283.

37 Also einschließlich der Handels-, Entwicklungs-, Nachbarschafts- und Erweiterungspolitik. 
befürworten das Ratssekretariat. ${ }^{38}$ Kommissionspräsident Barroso und der Hohe Vertreter Solana schlagen in einem gemeinsamen Papier eine dritte Möglichkeit vor. ${ }^{39}$ Alle Beamten, die sich (gleich ob abgeordnet oder permanent) zurzeit im Rat und in der Kommission mit Fragen der GASP und der ESVP befassen, sollen demnach in den Europäischen Auswärtigen Dienst übernommen werden. Offen ist zudem noch die künftige Rolle der Auslandsvertretungen der Kommission. Gleich in welche Lösung die seit Ende 2004 laufenden Bemühungen auch münden werden, die durch einen solchen Dienst hervorgerufene Verflechtung und die Praxis ständiger Zusammenarbeit , unter einem Dach “ würde sicherlich die außenpolitische Identität und die Kohärenz beträchtlich fördern.

Ein wesentliches Ziel des außen- und sicherheitspolitischen Handelns der Union ist die schrittweise Entwicklung einer auf den gemeinsamen Werten, Interessen und Erfahrungen beruhenden europäischen Sicherheitskultur, welche die nationalen Sicherheitskulturen der Mitgliedstaaten zumindest partiell überwindet und zu einer europäischen außen- und sicherheitspolitischen Identität beiträgt. ${ }^{40}$ Eine ganz praktische, von der Ratifizierung des VVE unabhängige Maßnahme zur Förderung dieses langfristigen Prozesses, die zugleich notwendig für die effektive Durchführung des EU-Krisenmanagements ist, ist eine gemeinsame Ausbildungspolitik. Erste Schritte in diese Richtung sind bereits eingeleitet worden. So wurden erste Trainingskurse zu zivilen und militärischen Aspekten des Krisenmanagements abgehalten und ein entsprechendes koordiniertes Konzept vom Rat in Auftrag gegeben. ${ }^{41}$ Das bereits in Thessaloniki im Juni 2003 betonte Ziel einer europäischen Sicherheitskultur dürfte durch die neue Europäische Akademie für Sicherheit und Verteidigung etwas näher gerückt sein. ${ }^{42}$ Gleichwohl müssen die Lehrinhalte der verschiedenen Ausbildungseinrichtungen gerade im Hinblick auf die Erfordernisse des zivil-militärischen Krisenmanagements optimal abgestimmt werden. ${ }^{43}$ Bei der gemeinsamen Ausbildung muss vor allen Dingen beachtet werden, dass die unterschiedlichen zivilen und militärischen Kulturen (Mentalitäten, Arbeitsmethoden, Normen) verstanden und zusammengeführt werden mit dem Ziel, die Synergie der GASP/ESVP zu erhöhen. ${ }^{44}$

Dazu könnten auch weitere institutionelle Anpassungen beitragen. So ist zu fragen, ob dem Außenminister der Union angesichts seiner umfangreichen Verpflichtungen nicht ein Stellvertreter zur Seite gestellt werden sollte. Dieser könnte die Sitzungen des PSK leiten und den Außenminister gegebenenfalls in anderen Angelegenheiten vertreten. Des Weiteren müsste das Konzept des Krisenmanagements reformiert werden. Da es fraglich ist, ob und wann der VVE ratifiziert wird, müsste bereits vorher der komplizierte Entscheidungsprozess vereinfacht werden. So wäre es schon ein Fortschritt, wenn an Stelle der bislang angefertig-

38 Elmar Brok, der Vorsitzende des Auswärtigen Ausschusses des EP führt zwei Argumente an: Die Wahrung der politischen Kontrolle über eine ansonsten verselbstständigte Einrichtung und die drohende Stärkung des intergouvernementalen Einflusses selbst bei Gemeinschaftsaufgaben. Vgl. Horst Bacia: Unabhängig oder im Dienst der Kommission?, in: Frankfurter Allgemeine Zeitung, 4.3.2005.

39 Vgl. Bacia: Unabhängig oder im Dienst der Kommission.

40 Grundlegend dazu Peter J. Katzenstein (Hrsg.): The culture of national security. Norms and identity in world politics, New York 1996.

41 Vgl. General Affairs and External relations Council: Conclusions, Brüssel, 17. November 2003, in: Antonio Missiroli (Hrsg.): From Copenhagen to Brussels. European defence: core documents, Chaillot Papers 67/2003, S. 260.

42 Vgl. European Council (Thessaloniki): Conclusions, in: Antonio Missiroli (Hrsg.): From Copenhagen to Brussels, S. 157.

43 Mittlerweile befassen sich fünf Einrichtungen mit GASP/ESVP-relevanter Ausbildung: Nationale Einrichtungen, das European Diplomatic Programme, das European Police College, das European Security and Defence College und das Trainingsprogramm für ziviles Krisenmanagement der Europäischen Kommission.

44 Vgl. Council of the European Union: Draft Final Training Report (FTR) of Training in ESDP, 7770/2/05, Brüssel 2005. 
ten drei strategischen Optionen eine gemeinsame strategische Option der ESVP (ESVP-SO) erarbeitet würde. Im Grunde genommen würde der mittlerweile im Militär übliche joint planning process auf den zivilen Bereich übertragen, um so eine effektivere, weil integrierte zivil-militärische Abstimmung zu erreichen. Diese Abstimmung einer ESVP-SO könnte entweder im Rahmen eines um ziviles Personal der GD E ergänzten Joint CAT erfolgen oder innerhalb der dem EUMS und der DG E überzuordnenden Zivil-Militärischen Zelle. Die ESVP-SO könnte als Grundlage für die Erstellung eines ESVP-Operationskonzepts durch die Zivil-militärische Zelle dienen und so für größere Kohärenz im Planungsverfahren sorgen. Erfordert die Lage eine sehr schnelle Reaktion, so sollten das Überspringen einzelner Planungsstufen, parallele Planung und ein größerer Handlungsspielraum für das PSK erwogen werden. Schließlich sei noch einmal an die Notwendigkeit erinnert, die Kompetenzen des Sonderbeauftragten zu stärken. Nur so kann die koordinierte Umsetzung der Operationspläne vor Ort verbessert werden. Der Sonderbeauftragte müsste also über echte Koordinierungskapazitäten verfügen. Dazu gehörte die möglichst frühe Ernennung von Vertretern der jeweiligen Operationsleiter (also des HoM, PolHoM, Head of Commission und OpCdr), die dem EUSR vor Ort zuarbeiten sowie die obligatorische Beteiligung aller Akteure vor Ort an einer Joint Coordination Group.

\section{Perspektiven}

Das zivil-militärische Krisenmanagement im Rahmen der ESVP ist noch sehr jung. Die ESVP wurde vor gut fünf Jahren ins Leben gerufen, die erste Operation begann vor zweieinhalb Jahren im Januar 2003. Seit dieser Zeit entwickeln sich die Strukturen, Verfahren, Ressourcen und Aktivitäten kontinuierlich, ohne dass bereits eine endgültige Form erkennbar ist. Ursache dafür sind neben der noch fehlenden Erfahrung nicht zuletzt die mangelnde Bereitschaft der Mitgliedstaaten weiter zu gehen, das komplizierte Governance-System der Europäischen Union und die noch zu schwache europäische Identität, oder anders ausgedrückt: das Fehlen eines gemeinsamen Leitbildes europäischer Außen- und Sicherheitspolitik. Diese Ursachen werden sich weder vollständig noch schnell abstellen lassen. Gleichwohl entwickelt sich das eigene Rollenverständnis und das institutionelle Setting des Krisenmanagers Europäische Union dynamisch. Die durch den Ausgang der Referenden in Frankreich und den Niederlanden bewirkte Krise muss sich nicht unbedingt negativ auf die GASP/ESVP auswirken, denn einige Reformen werden bereits umgesetzt und andere könnten auch ohne die Ratifizierung des VVE beschlossen werden. Die Verbesserung der inter- und intrainstitutionellen Kohärenz bleibt eine der großen Herausforderungen für ein effektiveres zivil-militärisches Krisenmanagement. Insbesondere die bisherige Zweiteilung des außenpolitischen Handelns müsste zu Gunsten einer verstärkten Vernetzung zwischen Kommission und dem GASP-Pfeiler überwunden werden. Doch darf dies nicht, wie bislang üblich, zu noch größerer Komplexität und Segmentierung führen. Vielmehr müssen angesichts der Realitäten des 21. Jahrhunderts Strukturen und Verfahren gefunden werden, die über die oben skizzierten Reformen und Reformvorschläge hinaus gehen. Zugleich muss dieser Prozess mit den Bürgerinnen und Bürgern der Union kommuniziert werden, um die relativ hohe Zustimmung zur GASP/ESVP auch im Einsatz aufrechtzuerhalten..$^{45}$ Nur so wird die Europäische Union ihren in der Europäischen Sicherheitsstrategie formulierten Anspruch verwirklichen können, als handlungsfähiger Akteur ein sicheres Europa in einer besseren Welt zu gestalten.

45 Vgl. dazu Wolfgang Wagner, Für Europa sterben? Die demokratische Legitimität der Europäischen Sicherheits- und Verteidigungspolitik, HSFK-Report 3/2004. 